\title{
INFLUENCE OF BARIUM DOPING ON MICROSTRAIN AND DIELECTRIC PROPERTIES OF THE LA2NIMnO6 DOUBLE PEROVSKITE
}

\author{
Barbosa, D. A. B. ${ }^{1}$; Almeida, R. M. ${ }^{2}$; Moreira, R. L. ${ }^{2}$; Paschoal, C. W. A. ${ }^{1, *}$ \\ ${ }^{1}$ Departamento de Física, Universidade Federal do Maranhão, Campus do Bacanga, São Luis, MA, Brazil. \\ 2 Departamento de Física, Universidade Federal de Minas Gerais, Belo Horizonte, Minas Gerais, Brazil \\ *paschoal.william@gmail.com
}

\begin{abstract}
Multiferroic are interesting materials that show coupling between at least two ferroic orders, usually magnetism and polarizability. $\mathrm{La}_{2} \mathrm{NiMnO}_{6}$ is one of the most studied ceramic bulk materials among multiferroics because presents magnetodielectric effect and is ferromagnet semiconductor near room temperature, but its intrinsic dielectric properties are scarcely investigated. In this investigation we doped $\mathrm{La}_{2} \mathrm{NiMnO}_{6}$ with different barium concentrations to observe the effects of structural strain variation in the intrinsic dielectric properties of $\mathrm{La}_{2} \mathrm{NiMnO}_{6}$. Ceramic samples were synthesized by standard solid state reaction and structural and vibrational characterization were performed by XRD and Infrared Spectroscopy. Rietveld Method and GSAS code were employed to refine the structure. Gervais and Piriou semi-quantum model was used to analyze the vibrational data. The results show that even the smallest degree of barium doping changes the phases ratio and decrease the strain in the lattice what favors high quality factors in the ceramic bulk. Large quality factors are usually related to high degrees of ordering in double perovskites which in $\mathrm{La}_{2} \mathrm{NiMnO}_{6}$ case means predominance of $\mathrm{Ni}^{2+}-\mathrm{O}-\mathrm{Mn}^{4+}$ superexchange bonds rather than $\mathrm{Ni}^{3+}-\mathrm{O}-\mathrm{Mn}^{3+}$ which is very desirable whereas superexchange bonds provides strong magnetism.
\end{abstract}

